\title{
Sincronía y diacronía en página web. Las organizaciones del IBEX35 y su ajuste a los modelos de comportamiento comunicativo empresarial de Grunig y Hunt
}

\author{
Alejandro TAPIA FrADE \\ Universidad Europea Miguel de Cervantes \\ alejandro.tapia.frade@gmail.com \\ Raquel CAerols Mateo \\ Universidad Antonio de Nebrija \\ rcaerols@nebrija.es
}

Recibido: 5 de febrero de 2014

Aceptado: 8 de septiembre de 2014

\begin{abstract}
Resumen
Este trabajo analiza la exposición en página web de información a través de la sala de prensa virtual, y sus posibilidades de retroalimentación. Con los resultados se hizo un análisis para considerar su ajuste a los modelos de comportamiento comunicativo empresarial de Grunig y Hunt. Los resultados ponen de manifiesto una gestión adecuada en cuanto a la exposición de información. Aunque también se puede hablar de presencia de sistemas de realimentación de información, se podría sugerir que su gestión es mejorable, al presentar una limitada frecuencia y capacidad expresiva. En consecuencia, se debe considerar la asimetría sistémica entre los sistemas de exposición (muy desarrollados) y realimentación (con un desarrollo más limitado para los públicos), que genera un sistema sincrónico y se ajusta al modelo de comportamiento bidireccional asimétrico.

Palabras clave: internet, IBEX35, sala de prensa, Grunig, Hunt.

\section{Synchrony and diachrony on websites. IBEX35 organizations and their adjustment to Grunig and Hunt's models of communicative behavior}

\begin{abstract}
This paper analyzes the exposition of information in websites through the virtual press room, and its possibility of feedback. With the analysis results given, we consider the adjustment to Grunig and Hunt's communicative behavior business models. The results demonstrates an appropriate management regarding the exposure of information. Although there is information feedback systems, it could be suggested that its management is could be improved, due a limited presence and expressive capability. Consequently, a structural asymmetry between systemic exposure systems (highly developed) and feedback (with a more limited development) should be considered, which generates a synchronous system and fits the behavior model to the bidirectional asymmetric one.
\end{abstract}

Keywords: internet, IBEX35, press room, Grunig, Hunt.

\section{Referencia normalizada}

TAPIA FRADE, Alejandro y CAEROLS MATEO, Raquel (2015): "Sincronía y diacronía en página web. Las organizaciones del IBEX35 y su ajuste a los modelos de comportamiento comunicativo empresarial de Grunig y Hunt". Estudios sobre el Mensaje Periodístico. Vol. 21, Núm. 2 (julio-diciembre), págs.: 913925. Madrid, Servicio de Publicaciones de la Universidad Complutense.

Sumario 1. Introducción. 2. Metodología. 3. Resultados; 3.1. La exposición de información: la sala de prensa; 3.2. La retroalimentación de información: la Web 2.0 y otras formas de comunicación; 3.3. El ajuste a los modelos de Grunig y Hunt. 4. Conclusiones. 5. Referencias bibliográficas. 


\section{Introducción}

La comunicación supone un activo estratégico esencial para cualquier organización que debe adaptarse a un público cada vez más informado.

En ese contexto resulta esencial la adopción de mecanismos de retroalimentación que permitan el retorno de información con dichos públicos de forma rápida y ágil con el fin de adaptarse con rapidez a las demandas de los mismos.

Internet constituye sin duda un canal de comunicación apto en términos expositivos, y especialmente interesante para labores realimentativas, al poseer algunas características críticas como la rapidez, la flexibilidad y la agilidad, que unido a una importante capacidad de segmentación dotan a las organizaciones de posibilidades antes no disponibles. Todavía más, con el uso de la Web 2.0 esas posibilidades de retroalimentación informativa se ven sustancialmente incrementadas, hasta el punto de la construcción cooperativa de discurso en medios digitales.

Es un medio que goza de una amplia cobertura, estimada en el 64\%, siendo además el soporte que más crece en audiencia y con una tendencia igualmente creciente (AIMC, 2013:2). Además, la frecuencia de conexión pone de manifiesto un claro uso diario y en crecimiento de internet, lo que añade cotidianeidad al hecho de conectarse, normaliza el hecho y por tanto propicia aún más su generalización (AIMC, 2013:5).

Tanto es así, que se ha llegado a sugerir la existencia de una sociedad basada en el entorno red, la sociedad red (Castells, 2000:1), interactiva, que en opinión de Cely Álvarez (1999) y Gobbi (2010) altera de hecho los tradicionales roles de emisor y receptor de información, en los últimos tiempos especialmente agudizado por la presencia de la llamada Web participativa -Web 2.0-.

En ese entorno virtual, la sala de prensa constituye un potente canal de difusión de noticias y relación con los medios, siendo por tanto un activo fundamental para las organizaciones (Peñalva, 2004: 365). Además, crea sinergias organizativas al sustituir este canal cierta cantidad de recursos necesarios para la realización cotidiana de la tarea periodística.

Literatura al respecto no falta. Así, Pineda Martínez (2013:189) destacó que la comunicación en Internet ha comenzado a representar una preocupación para las compañías del IBEX35, si bien Internet no resultó ser un recurso bien gestionado para crear relaciones con los grupos de interés.

Un estudio anterior específico sobre exposición de información en salas de prensa e interacción a través de la web 2.0 destacó que las empresas del IBEX35 atienden los contenidos publicados en sus salas de prensa virtuales, mediante la publicación periódica de nuevas informaciones y la elaboración de los mismos más allá de las notas de prensa y los comunicados. Respecto de la Web Participativa, concluyeron que el uso de herramientas 2.0 presenta un balance global negativo, ya que solamente una minoría utiliza dichas herramientas. Así, el uso de blogs corporativos es bajo y las redes sociales tampoco están implantadas en los planes de comunicación de estas organizaciones (Sánchez Pita y Rodríguez Gordo, 2010:13).

En definitiva, este trabajo trata sobre la sincronía y diacronía en el proceso comunicativo de las empresas del IBEX35 en sus páginas Web, con el objetivo de determinar si está adecuadamente estructurado, o si por el contrario se dan deficiencias 
importantes tanto en lo relativo a los contenidos expuestos como a los recursos realimentativos utilizados.

Posteriormente, se analizan los resultados para considerar su ajuste a los modelos de comportamiento comunicativo empresarial de Grunig, lo que supone considerar el estadio evolutivo en términos de comunicación en que se encuentran estas compañías.

\section{Metodología}

A fin de conocer en qué situación se encuentran las empresas del IBEX35 en lo relativo a la exposición y retroalimentación de información por Internet y su consecuente ajuste a los modelos de comportamiento comunicativo de Grunig, se decidió el uso de la técnica del análisis de contenido.

El análisis se limita únicamente a la información emitida en la versión web de escritorio de las compañías del IBEX35, no ampliándose a otras empresas participadas, filiales o pertenecientes al mismo grupo empresarial.

Así, se realizó una plantilla de análisis compuesta por 64 variables que estructuran e ilustran las posibilidades comunicativas que ofrecen dichas organizaciones en cuanto a comunicación en web.

La plantilla se estructura en primer lugar a dos niveles: valorando la presencia de elementos que posibilitan exposición y retroalimentación informativa. Es decir, si en la página Web se articulan mecanismos que posibilitan no sólo la mera exposición de información sino además la posibilidad de intercambio de información para la organización (feedback informativo) y para los públicos (atención personalizada a los distintos públicos).

En la exposición de información del sitio Web se consideró la existencia de un centro o sección destinada específicamente a la relación con sus públicos donde se vuelca contenido -tales como noticias, notas de prensa, boletines, etc.- relacionado con la organización o su actividad en tono periodístico. Nos referimos a la existencia de una sala de prensa virtual, cuyos antecedentes se sitúan en los antiguos gabinetes de prensa (Soria Ibáñez, 2009).

También se valoró si en la comunicación emitida se empleaba únicamente texto o si se aprovechaban las cualidades del medio y se incluía fotografía, audio y vídeo.

Por último, para determinar las posibilidades realimentativas de los sitios Web se valoró si constaba email, formularios para pedir información y posibilidad de comentar, valorar o escribir sobre la organización y su actividad. Finalmente, se investigó la existencia de formas de comunicación con altas posibilidades de generación de simetrías comunicativas como chats, blogs, etc.

Se usó la misma escala para determinar no sólo presencia o ausencia de los elementos -12-, sino también si los mismos se encuentran en la sección de sala de prensa o en otro lugar de la página web. Así, dicha escala constaba de cuatro elementos que transitaban entre la presencia en el lugar más acertado para dicho contenido ("Está, en la sala de prensa") a la ausencia del elemento ("No está"), pasando por dos estadios intermedios: "Está, pero en sección de información corporativa" (menos acertado a nuestro parecer que la sala de prensa, pero más acertado que en otra sección distinta de ambas) y "Está, pero fuera de ambas secciones" (la de sala de prensa y la información corporativa). 
El análisis de fiabilidad de dicha escala mediante el estadístico Alfa de Cronbach obtuvo un resultado de 0,716 , que aun siendo bajo, resulta válido considerando que el propósito es únicamente la investigación.

Se ejecutó dicha plantilla de análisis sobre los sitios Web de las 35 organizaciones constituyentes del índice bursátil IBEX35. La recogida de información se efectuó durante el mes de enero de 2014.

Por tanto, nos encontramos ante el análisis de la totalidad del universo de estudio, o lo que es lo mismo, un estudio por censo. Dicho estudio fue posible dado el limitado tamaño y amplia disponibilidad del universo, que permitió la recogida de información en un corto espacio de tiempo, consideración ésta imprescindible ante un estudio de tipo transversal como el que nos ocupa.

Los resultados se estratificaron según la clasificación sectorial vigente de Bolsas y Mercados Españoles (BME). Dicho índice tiene vigencia desde el 1 de enero de 2005.

\section{Resultados}

Como inicio a la cuestión, hay que señalar que todas las organizaciones constituyentes del índice bursátil IBEX35 tienen página web, y que ésta estaba completamente operativa en el momento de la realización del análisis. Otra cuestión es el alcance geográfico que se haya deseado para tales páginas, que podría interpretarse en función de los lenguajes en que esté disponible dicha página web.

En el caso que nos ocupa, se puede señalar una tendencia general ${ }^{1}$ acusada al uso del español y el inglés ( $68,57 \%$ de los casos), y en menor medida, al uso de un tercer lenguaje $(28,57 \%)$, lo que podría considerarse apropiado, teniendo en cuenta la magnitud de las organizaciones y consecuentemente la amplitud de sus operaciones.

\subsection{La exposición de información: la sala de prensa}

La sala de prensa permite, con gran plasticidad de formatos, la exposición de todo tipo de información a todos los públicos, pero con especial atención a otros difusores de información, los medios de comunicación. La mencionada plasticidad permite la adaptación de contenidos a todo tipo de medios, que pueden acceder a cualquier información de la organización con rapidez, característica ésta fundamental para los medios de comunicación.

Los datos ponen de manifiesto que la totalidad de las mismas poseen una sala de prensa virtual en su página web, que además se sitúa en un lugar preeminente de la página web, a tan sólo un clic de la página de inicio, no siendo necesario en ningún caso analizado registro para acceder.

Los nombres más comunes dados a esta sección son Sala de prensa (42,5\% de los casos), Prensa (20\%) y Comunicación $(11,5 \%)$ y Noticias $(11,4 \%)$.

1 El coeficiente de significación para el estadístico Chi Cuadrado de Pearson en la tabla de contingencia es de 0,298 , por lo que no cabe señalar relación entre el sector de las organizaciones y el uso de los lenguajes por parte de las mismas. 
En lo referente al contenido de la sala de prensa, en este trabajo se consideró, por un lado, la presencia y periodicidad de noticias, recortes de prensa, artículos de investigación, y newsletter y, por otro, su ubicación considerando la escala antes mencionada en la metodología.

Todas las organizaciones analizadas disponían en el momento del análisis de noticias, y siempre ubicadas en la sala de prensa. Adicionalmente, la mayoría (85,7\% del total) ofrecen las noticias relevantes en la página de inicio, lo que hace sugerir un alto nivel de importancia estratégica concedida a este particular. Dichas noticias aparecen en general (94,3\% de los casos) clasificadas por fecha de emisión, sin haberse encontrado diferencias significativas entre sectores ${ }^{2}$.

Respecto de la antigüedad de las mismas, en todos los sectores se encontraron noticias con más de tres años de antigüedad, salvo en los sectores de Servicios de consumo y Tecnología y telecomunicaciones (en éstas, sólo el 50\% de las empresas tenían noticias con más tres años de antigüedad).

Además, se observó que la frecuencia de actualización de las noticias debe considerarse en general ${ }^{3}$ adecuada., de acuerdo con los datos expuestos en tabla 1.

Tabla 1. ¿Con qué frecuencia se actualizan las noticias? (\%). Fuente: Elaboración propia.

\begin{tabular}{|c|c|c|c|c|c|}
\hline \multicolumn{2}{|c|}{ ¿Cada cuánto tiempo se actualizan? } & $\begin{array}{l}\text { Todos los } \\
\text { días }\end{array}$ & $\begin{array}{l}\text { Varias veces a } \\
\text { la semana }\end{array}$ & $\begin{array}{l}\text { Una vez a la } \\
\text { semana }\end{array}$ & $\begin{array}{l}\text { En periodos } \\
\text { superiores }\end{array}$ \\
\hline \multirow{6}{*}{$\begin{array}{l}\text { Sector de } \\
\text { actividad }\end{array}$} & Petróleo y energía & & 50,00 & 50,00 & \\
\hline & $\begin{array}{l}\text { Materiales básicos, industria y } \\
\text { construcción }\end{array}$ & & 55,56 & 11,11 & 33,33 \\
\hline & Bienes de consumo & & & 25,00 & 75,00 \\
\hline & Servicios de consumo & 25,00 & 25,00 & & 50,00 \\
\hline & Servicios financieros e inmobiliarias & 12,50 & 62,50 & 12,50 & 12,50 \\
\hline & Tecnología y telecomunicaciones & 50,00 & & & 50,00 \\
\hline \multicolumn{2}{|r|}{ Total } & 11,43 & 40,00 & 17,14 & 31,43 \\
\hline
\end{tabular}

No obstante, sería deseable que el sector de Bienes de consumo, y en menor medida los sectores de Servicios de consumo y Tecnología y telecomunicaciones mejoraran sus registros al respecto.

Dichas noticias van acompañadas mayoritariamente de fotografías $(65,71 \%$ de los casos) y material audiovisual (57,14\% del total, lo que sugiere cierto grado de planificación y elaboración de las noticias, salvo para los sectores de Bienes de consumo (apenas un $25 \%$ de ellos acompañan con fotografía o material audiovisual a la noticia) y en menor medida el de Materiales básicos (55,56\% de empresas de este sector acompaña a la noticia con fotografía, y solo un tercio la acompaña de material audiovisual) $)^{4}$.

2 El coeficiente de Significación de Chi Cuadrado hallado fue de 0,469.

${ }^{3}$ Estadísticamente no pudieron afirmarse diferencias significativas en función del sector e actividad. El coeficiente de significación de Chi Cuadrado fue de 0,052 $(>0,050)$

${ }^{4}$ No obstante, dichas diferencias no justifican una diferencia estadística significativa general de los elementos analizados (el coeficiente de significación de Chi Cuadrado para fotografía fue de 0,220 y el de material audiovisual fue de 0,214 , en ambos casos $>0,05$ ) 
No obstante, la fotografía ${ }^{5}$ y el material audiovisual también se ubica en otros sitios distintos de la página web, en muchos casos en la sección de información corporativa $(71,42 \%$ del total en el caso de la fotografía, y $51,43 \%$ en el caso del vídeo) -mención aparte requieren los logotipos, que se encuentran en la sala de prensa en un $52,94 \%$ de los casos, considerándose por tanto más recurso periodístico que elemento corporativo-. Además, el material audiovisual suele también tener presencia en forma de canal en la red social YouTube (el 57,14\% de las empresas del IBEX35 tienen canal de youTube).

Otro elemento analizado fueron los recortes de prensa. En este caso hay que señalar un comportamiento general ${ }^{6}$ pobre (apenas el $22,86 \%$ de las empresas los incluyen), poniendo de relieve que éste es un recurso en desuso. Dentro de este nivel pobre general, puede apreciarse cierto uso (50\% de los casos) en los sectores de Servicios financieros y Tecnología y telecomunicaciones (en igual proporción), en cuyo caso se ubican siempre en la press room o sala de prensa.

Los artículos de opinión e investigación son muy comunes (los tienen el 97,14\% de los casos analizados) en todos los sectores ${ }^{7}$, y en todos los casos se ubican en la sala de prensa.

Otra elemento relevante para la difusión de información es sin duda el newsletter, o boletín periódico, que de acuerdo con los datos aportados en tabla 2, es un elemento presente en aproximadamente la mitad de los casos, si bien destaca su ausencia en el sector de Bienes de consumo y su presencia minoritaria en el de Servicios de consumo ${ }^{8}$. La suscripción es necesaria de forma minoritaria ( $21,1 \%$ de los casos) su periodicidad suele ser mensual o en periodos superiores $(85,7 \%$ de los casos) y el medio habitual de adquisición suele ser por descarga desde la página web (84,6\% de los casos)

Tabla 2. Presencia Newsletter y buscador específico de noticias (\%).

Fuente: Elaboración propia

\begin{tabular}{|l|l|r|r|}
\hline \multirow{2}{*}{ ¿Existe la posibilidad de suscribirse a boletines periódicos? } & \multicolumn{1}{l|}{$\begin{array}{l}\text { Si, en la Sala de } \\
\text { Prensa }\end{array}$} & \multicolumn{1}{c|}{ No } \\
\hline \multirow{4}{*}{$\begin{array}{l}\text { Sector de } \\
\text { actividad }\end{array}$} & Petróleo y energía & 50,00 & 50,00 \\
\cline { 2 - 4 } & Materiales básicos, industria y construcción & 66,67 & 33,33 \\
\cline { 2 - 4 } & Bienes de consumo & & 100,00 \\
\cline { 2 - 4 } & Servicios de consumo & 25,00 & 75,00 \\
\cline { 2 - 4 } & Servicios financieros e inmobiliarias & 87,50 & 12,50 \\
\cline { 2 - 4 } & Tecnología y telecomunicaciones & 75,00 & 25,00 \\
\hline \multicolumn{2}{|c|}{ Total } & 57,14 & 42,86 \\
\hline
\end{tabular}

5 Sin diferencias estadísticamente significativas por sectores (el valor del coeficiente de significación para la fotografía fue de 0,214 y de 0,189 para el material audiovisual)

6 El coeficiente de Significación de Chi Cuadrado hallado fue de $0,145(>0,05)$, por lo que no pueden concluirse desigualdades estadísticas significativas por sectores.

7 En este caso el Coeficiente de Significación de Chi Cuadrado fue de 0,704 $(>0,05)$.

8 El Coeficiente de Significación de Chi Cuadrado fue de 0,051 $(>0,050)$, por lo que prácticamente pueden señalarse diferencias significativas por sectores. 


\begin{tabular}{|c|l|r|r|}
\hline \multirow{4}{*}{ ¿Hay un buscador específico de noticias, artículosy hemeroteca? } \\
\hline \multirow{4}{*}{$\begin{array}{l}\text { Sector de } \\
\text { actividad }\end{array}$} & Petróleo y energía & 100,00 & \\
\cline { 2 - 4 } & Materiales básicos, industria y construcción & 66,67 & 33,33 \\
\cline { 2 - 4 } & Bienes de consumo & 100,00 & \\
\cline { 2 - 5 } & Servicios de consumo & 25,00 & 75,00 \\
\cline { 2 - 5 } & Servicios financieros e inmobiliarias & 87,50 & 12,50 \\
\cline { 2 - 5 } & Tecnología y telecomunicaciones & 50,00 & 50,00 \\
\hline \multicolumn{2}{|c|}{ Total } & 74,29 & 25,71 \\
\hline
\end{tabular}

Un aspecto fundamental en la página web es la facilidad de búsqueda de sus distintos contenidos. La potencialmente elevada cantidad de información en Web no sólo requiere una adecuada estructuración de la misma, sino también la disposición de herramientas que simplifiquen la búsqueda de cierta información entre todas las restantes. Este trabajo consideró la existencia de dichas herramientas de búsqueda en dos posiciones: a nivel de contenido general, y a nivel específico de noticias, hemeroteca, artículos de investigación y recortes de prensa. Los datos muestran la presencia general de un buscador especializado en contenido noticioso (superior al 74,29\% en términos generales, aunque con la excepción del sector Servicios de consumo, donde su presencia es minoritaria) y también de un buscador a nivel general (el 73,23\% de organizaciones disponen de él).

\subsection{La realimentación de información: la Web 2.0 y otras formas de comunica- ción}

La realimentación de información es sin duda una cuestión básica para cualquier organización que pretenda operar en el mercado de forma mínimamente eficiente, ya que sin ella no sería posible atender las demandas de los mercados, cada vez más dinámicos. Además, la adopción de un modelo simétrico de relación entre una organización y sus públicos requiere de dicha bidireccionalidad en la comunicación, además de proporcionalidad entre la intensidad de exposición y realimentación de información.

Por ello esta investigación tuvo en cuenta el estudio de elementos tradicionales de retroalimentación comunicativa como los formularios en Web, emails o teléfonos y otros típicos de la Web 2.0 como los blogs, la posibilidad de valorar o comentar noticias u opiniones, sindicación de noticias por RSS, los chats, redes sociales, canales de YouTube e Instagram etc.

Respecto a los métodos tradicionales de comunicación, emails y teléfonos son elementos presentes de forma generalizada las web en todas las organizaciones. Sin embargo, no sucede lo mismo en el caso de formularios de reclamaciones y contacto, cuya presencia es minoritaria, tal y como apreciarse en la tabla posterior. 
Tabla 3. Presencia formulario de sugerencias (\%). Fuente: Elaboración propia.

\begin{tabular}{|c|c|c|c|c|}
\hline \multicolumn{2}{|c|}{ ¿Hay un formulario de sugerencias? } & \multirow{2}{*}{\begin{tabular}{|r|}
$\begin{array}{l}\mathrm{Si} \text {, en la sección } \\
\text { de Información } \\
\text { Corporativa. }\end{array}$ \\
33,34 \\
\end{tabular}} & \multirow{2}{*}{\begin{tabular}{|l}
$\begin{array}{l}\mathrm{Si} \text {, fuera de la Sala } \\
\text { de Prensa y la Info. } \\
\text { Corporativa }\end{array}$ \\
33,33
\end{tabular}} & \multirow{2}{*}{$\begin{array}{l}\text { No } \\
33,33\end{array}$} \\
\hline \multirow{6}{*}{$\begin{array}{l}\text { Sector de } \\
\text { actividad }\end{array}$} & Petróleo y energía & & & \\
\hline & $\begin{array}{l}\text { Materiales básicos, industria y } \\
\text { construcción }\end{array}$ & 11,11 & 33,33 & 55,56 \\
\hline & Bienes de consumo & & 75,00 & 25,00 \\
\hline & Servicios de consumo & & & 100,00 \\
\hline & Servicios financieros e inmobiliarias & 14,29 & 14,29 & 71,42 \\
\hline & Tecnología y telecomunicaciones & 50,00 & 25,00 & 25,00 \\
\hline \multicolumn{2}{|r|}{ Total } & 17,65 & 29,41 & 52,94 \\
\hline
\end{tabular}

Además, no encontró ningún caso en que fuera ubicada en la sala de prensa.

La posibilidad de comentar o valorar noticias no ofrece mejores perspectivas. En ningún caso se podían comentar noticias, y tan sólo el 5,7\% de los casos analizados permitía la valoración. En conclusión, estos datos ponen de manifiesto la inexistencia sistemática de estos elementos.

Los datos analizados para nuevas formas de comunicación ofrecen una visión más optimista de estas organizaciones, aunque todavía podría calificarse de insuficiente, tal y como puede apreciarse en la tablas posteriores.

Así, la presencia de blogs manifiesta ciertas diferencias ${ }^{9}$ respecto de los sectores analizados. Su presencia es notoria en el sector de Bienes de consumo (con presencia $50 \%$ de los casos) y mayoritaria en el sector de Tecnología y telecomunicaciones (75\% de los casos). Su ubicación preferente de este elemento es la sala de prensa -salvo en los sector de Petróleo y energía y Tecnología y telecomunicaciones-, y los post publicados pueden comentarse ( $80 \%$ de los casos) pero no valorarse $(70 \%)$, actividad esta última que parece reservada para las redes sociales

Tabla 4. Items de realimentación comunicativa. Presencia (\%) (I).

Fuente: Elaboración propia.

\begin{tabular}{|c|l|r|r|r|r|}
\hline \multicolumn{2}{|c|}{ ¿Existe un blog en la web? } & $\begin{array}{l}\text { Si, en la } \\
\text { Sala de } \\
\text { Prensa }\end{array}$ & $\begin{array}{l}\text { Si, en la sección } \\
\text { de Info. } \\
\text { Corporativa. }\end{array}$ & $\begin{array}{l}\text { Si, pero } \\
\text { fuera de } \\
\text { ambas }\end{array}$ & \multicolumn{1}{c|}{ No } \\
\hline \multirow{5}{*}{$\begin{array}{l}\text { Sector de } \\
\text { actividad }\end{array}$} & Petróleo y energía & & & 83,67 & \\
\cline { 2 - 6 } & $\begin{array}{l}\text { Materiales básicos, industria y } \\
\text { construcción }\end{array}$ & 22,22 & & & 77,78 \\
\cline { 2 - 6 } & Bienes de consumo & & & & 100,00 \\
\cline { 2 - 6 } & Servicios de consumo & 25,00 & & 25,00 & 50,00 \\
\cline { 2 - 6 } & Servicios financieros e inmobiliarias & 25,00 & & 75,00 \\
\cline { 2 - 6 } & Tecnología y telecomunicaciones & 25,00 & 25,00 & 25,00 \\
\hline \multicolumn{2}{|c|}{ Total } & 17,15 & 5,71 & 71,43
\end{tabular}

9 Dichas diferencias no son estadísticamente significativas (el coeficiente de significación para el estadístico Chi Cuadrado fue de 0,345) 


\begin{tabular}{|c|c|c|c|c|c|}
\hline \multicolumn{6}{|c|}{ ¿Existe la suscripción a noticias por RSS? } \\
\hline \multirow{6}{*}{$\begin{array}{l}\text { Sector de } \\
\text { actividad }\end{array}$} & Petróleo y energía & 50,00 & 25,00 & & 25,00 \\
\hline & $\begin{array}{l}\text { Materiales básicos, industria y } \\
\text { construcción }\end{array}$ & 40,00 & & & 60,00 \\
\hline & \begin{tabular}{|l|} 
Bienes de consumo \\
\end{tabular} & & & & 100,00 \\
\hline & \begin{tabular}{|l|} 
Servicios de consumo \\
\end{tabular} & 25,00 & & 25,00 & 50,00 \\
\hline & Servicios financieros e inmobiliarias & 62,50 & & 12,50 & 25,00 \\
\hline & Tecnología y telecomunicaciones & 50,00 & 25,00 & & 25,00 \\
\hline \multicolumn{2}{|r|}{ Total } & 41,37 & 6,90 & 6,90 & 44,83 \\
\hline
\end{tabular}

La suscripción de noticias mediante RSS también presenta ciertas diferencias ${ }^{10}$ según el sector considerado. Su presencia es mayoritaria en todos los sectores salvo el de Bienes de consumo y el de Materiales básicos, industria y construcción. Su ubicación preferente, como parece más lógico, es la sala de prensa.

La posibilidad enviar contenido a las redes sociales ${ }^{11}$ también sostiene diferencias por sectores ${ }^{12}$, tal y como apreciarse en tabla 5. Así, mantiene un nivel muy alto de presencia en los sectores de Petróleo y energía, Servicios financieros e inmobiliarios y Tecnología y telecomunicaciones, y muy bajo en los sectores de Bienes de consumo y Servicios de consumo.

La presencia de canales de YouTube defiere de forma significativa ${ }^{13}$ según el sector analizado: En el caso de los sectores de Bienes y Servicios de consumo es minoritaria, en el de Materiales básicos es la presencia es de aproximadamente la mitad de los casos analizados, y en el resto de sectores la presencia de canales de YouTube es mayoritaria.

Finalmente, hay que señalar que el uso de Instagram es minoritario en todos los sectores ${ }^{14}$, aunque se aprecia cierto uso de los sectores de Servicios financieros e inmobiliarias y el de Bienes de consumo.

Tabla 5. Items de realimentación comunicativa. Presencia (\%) (II). Fuente: Elaboración propia

\begin{tabular}{|c|l|r|r|}
\hline \multicolumn{2}{|c|}{ ¿Se puede enviar contenido a redes sociales? } & $\mathrm{Si}$ & \multicolumn{1}{c|}{ No } \\
\hline \multirow{5}{*}{$\begin{array}{l}\text { Sector de } \\
\text { actividad }\end{array}$} & Petróleo y energía & 100 & \\
\cline { 2 - 4 } & Materiales básicos, industria y construcción & 55,56 & 44,44 \\
\cline { 2 - 4 } & Bienes de consumo & & 100 \\
\cline { 2 - 4 } & Servicios de consumo & 25 & 75 \\
\cline { 2 - 4 } & Servicios financieros e inmobiliarias & 100 & \\
\cline { 2 - 4 } & Tecnología y telecomunicaciones & 75 & 25 \\
\hline \multicolumn{2}{|c|}{ Total } & 65,71 & 34,29 \\
\hline
\end{tabular}

${ }^{10}$ Dichas diferencias tampoco fueron estadísticamente significativas (en este caso el mencionado coeficiente de significación fue de 0,381 )

${ }^{11}$ Fundamentalmente Facebook, twitter, flickr y Pinterest

${ }^{12}$ En este caso las diferencias por sectores halladas fueron estadísticamente significativas a un $95 \%$ de probabilidad (el valor del coeficiente de significación para el estadístico Chi Cuadrado fue de 0,02 -menor de 0,05-)

${ }^{13}$ En este caso, el coeficiente de significación muestra un valor de 0,013, por lo que es significativo con una probabilidad del $95 \%(<0,05)$.

${ }^{14}$ El coeficiente de significación en la prueba Chi Cuadrado arroja un resultado de 0,278. 


\begin{tabular}{|c|c|c|c|}
\hline \multicolumn{4}{|c|}{ ¿Hay canal en youTube? } \\
\hline \multirow{6}{*}{$\begin{array}{l}\text { Sector de } \\
\text { actividad }\end{array}$} & Petróleo y energía & 66,67 & 33,33 \\
\hline & Materiales básicos, industria y construcción & 44,44 & 55,56 \\
\hline & Bienes de consumo & & 100 \\
\hline & Servicios de consumo & 25 & 75 \\
\hline & Servicios financieros e inmobiliarias & 100 & \\
\hline & Tecnología y telecomunicaciones & 75 & 25 \\
\hline \multicolumn{2}{|r|}{ Total } & 57,14 & 42,86 \\
\hline \multicolumn{4}{|c|}{ ¿Tienen perfil en Instagram? } \\
\hline \multirow{6}{*}{$\begin{array}{l}\text { Sector de } \\
\text { actividad }\end{array}$} & Petróleo y energía & & 100 \\
\hline & Materiales básicos, industria y construcción & 11,11 & 88,89 \\
\hline & \begin{tabular}{|l|} 
Bienes de consumo \\
\end{tabular} & 25 & 75 \\
\hline & Servicios de consumo & & 100 \\
\hline & Servicios financieros e inmobiliarias & 37,5 & 62,5 \\
\hline & Tecnología y telecomunicaciones & & 100 \\
\hline \multicolumn{2}{|r|}{ Total } & 14,29 & 85,71 \\
\hline
\end{tabular}

\subsection{El ajuste a los modelos de comportamiento comunicativo empresarial de Gru- nig y Hunt}

Grunig y Hunt establecieron en 1984 cuatro modelos de actuación en comunicación institucional -los modelos de agente de prensa, información pública, bidireccional asimétrico y bidireccional simétrico- que además relacionaron con estadios evolutivos en comunicación (Grunig y Hunt, 1984).

La distinción entre modelos, y por tanto la articulación de la diferencia evolutiva, se señaló en función esencialmente de dos ejes: la dirección y el propósito. La primera distinguía entre modelos unidireccionales y bidireccionales en función de la existencia o no de mecanismos de realimentación informativa y la segunda ahondaba en la simetría o asimetría de dicha relación.

En aplicación de estas variables, los dos primeros modelos-agente de prensa e información pública- son unidireccionales (no hay por tanto mecanismos de realimentación implantados en ellos) La diferencia se basaba en que mientras en el primero la comunicación se basaba en el engaño, en la propaganda en su sentido más peyorativo, el segundo el propósito era la información veraz: el receptor no participa de la información pero la conoce.

El tercer y el cuarto modelo -bidireccional asimétrico y bidireccional simétrico- son bidireccionales, habiendo por tanto mecanismos de realimentación de información implantados. La diferencia entre ambos estriba en la sincronía (o asimetría) o diacronía (o simetría) del diálogo. El sistema sincrónico busca la únicamente el beneficio empresarial, es decir, no constituye un diálogo cierto, sino que busca la investigación de audiencias -relacionado con el modelo bidireccional asimétrico-. El sistema diacrónico, en cambio, busca el diálogo, el beneficio mutuo en la relación organización público, asumiendo por tanto la posibilidad de cambio en la organización, no sólo en el público. 
Se consideró el ajuste a los modelos de Grunig y Hunt exclusivamente en función de su comunicación en página web. Por tanto, no se pretende en este trabajo señalar el estadio comunicativo total en que se encuentran las organizaciones del IBEX35, sino simplemente considerar ese estadio comunicativo únicamente en cuanto a la comunicación página web.

En este trabajo consideramos la simetría informativa en cuanto equilibrio entre los sistemas de exposición -destinados a la provisión de información a sus públicos- y realimentación -destinados a escuchar las demandas de los públicos-.

En consecuencia, cuanto mayor el equilibrio -en presencia y potencia- entre ambos, mayor probabilidad de simetría sistémica en el sistema comunicativo organizacional y en la información que por él fluye, y consecuentemente más probabilidad de diacronía en la relación organización - públicos.

Así, en cuanto exposición de información, cabe señalar un buen desempeño general. La presencia de la sala de prensa es incuestionable, y también lo es la presencia de noticias, artículos de opinión e investigación que se actualizan con gran frecuencia, que van acompañadas de vídeo y fotografía y que con gran frecuencia están disponibles en la página de inicio. Además, se han incorporado sistemas de búsqueda a nivel general y a nivel de noticias para conseguir accesibilidad en dicha información. Finalmente, la exposición de información corporativa también puede calificarse en general de adecuada.

No obstante, los sistemas de realimentación, si bien son un hecho y su presencia es mayoritaria, no gozan de tan adecuada gestión. Así, no existe la posibilidad de comentar o valorar las noticias que con tanta dedicación se exponen y la presencia de formularios para escribir comentarios sólo es un hecho en la mitad de las webs analizadas. Los blogs están presentes en únicamente el $25 \%$ de los casos, si bien cuando se dan permiten el comentario del post ( $80 \%$ de los casos, aunque con previa censura por parte del administrador), pero no su valoración (únicamente el $30 \%$ de los casos). Las redes sociales se usan para compartir noticias mayoritariamente (65\% de los casos), aunque en menor medida se usa YouTube (57\% de los casos) e Instagram apenas está presente en el 14\% de los casos. Finalmente, los sistemas clásicos de diálogo (teléfono y email) están en todos los casos presentes.

Por tanto, aunque se puede hablar de presencia de sistemas de realimentación de información, se podría sugerir que su gestión es manifiestamente mejorable, al presentar una limitada frecuencia y capacidad expresiva.

En consecuencia, se debe considerar la asimetría sistémica entre los sistemas de exposición (muy desarrollados) y realimentación (con un desarrollo más limitado para los públicos), que genera un sistema sincrónico con un marcado fin de recogida de información para investigación de audiencias.

Por tanto, se puede sugerir que en lo relativo a la comunicación por página web, las empresas del IBEX35 se ajustan al modelo de comportamiento bidireccional asimétrico.

\section{Conclusiones}

La presencia en internet de las empresas del IBEX35 es un hecho incuestionable, como también lo es la presencia de una sala de prensa en la misma. Un estudio similar, aun- 
que sobre las organizaciones del ranking Fortune 500, puso de manifiesto el incremento de contenidos diseñados para los periodistas, en línea con lo mostrado en este trabajo (Pettigrew y Reber, 2010: 404-428), lo que supone cierta evolución desde 2003, cuando un estudio sobre las mismas organizaciones puso de manifiesto la minoritaria presencia de este recurso en la web (Callison, 2003: 29-41).

Además, se puede destacar un buen desempeño general de los sistemas de exposición de información, tanto en estructura (la presencia de la sala de prensa virtual es un hecho incuestionable) como en contenidos (la adecuación de las noticias y contenidos corporativos a la web es manifiesta).

No obstante, no puede señalarse lo mismo de los sistemas de realimentación de información, que en esencia no parecen destinados a la generación de diálogo dada la marcada limitación expresiva impuesta a los públicos (no se permiten comentarios a noticias, no abundan los formularios, la presencia de blogs donde hacer comentarios -eso sí, previa censura del administrador- apenas llega al 25\%, etc.), sino a la mera recogida de información con fines de investigación de audiencias.

Por consiguiente, puede sugerirse cierto grado de asimetría comunicativa -a nivel sistémico- que sitúa a estas organizaciones en el estadio bidireccional asimétrico en la teoría de Grunig y Hunt.

Las limitaciones más importantes de este trabajo se relacionan con el contenido mismo del discurso. Es decir, se estudia qué presencia tienen ciertos contenidos y estructuras comunicativas, pero no se estudia ni el contenido del discurso ni los efectos que dicho discurso genera en los públicos.

El análisis de la comunicación se hace exclusivamente a la versión de web corporativa de escritorio, no incluyendo por tanto versiones de Smartphone y Tablet. Además, el análisis se limita a las webs corporativas de los grupos empresariales, no ampliándose a otras empresas participadas, filiales o pertenecientes al mismo grupo empresarial.

Futuras líneas de investigación podrían incluir tanto otras versiones (web móvil, tabletas, apps, etc.) de comunicación por internet, como otras webs de empresas filiales o participadas. Además, podría ser interesante el estudio de entornos competitivos similares.

\section{Referencias bibliográficas}

AIMC (2013): EGM. Estudio de la audiencia de internet. http://www.aimc.es [Consulta: 14 de diciembre de 2013].

CASTELLS, Manuel (2000): "Internet y la sociedad red". Conferencia de Presentación del Programa de Doctorado sobre la Sociedad de la Información y el Conocimiento. Barcelona. Universitat Oberta de Catalunya.

CALLISON, Coy (2003): "Media relations and the Internet: how Fortune 500 company web sites assist journalists in news gathering". Public Relations Review, 29 (1), pp. 29-41. http://dx.doi.org/10.1016/S0363-8111(02)00196-0

CELY ÁLVAREZ, Adriana (1999): "Elementos para caracterizar los nuevos medios de comunicación”. Revista Latina, 19. Tenerife. Universidad de La Laguna. En: 
http://www.ull.es/publicaciones/latina/a1999fjl/68acely.htm [Consulta: 12 de diciembre de 2013].

GOBBI, Jorge (2010): "Medios, Internet y periodismo: del modelo unidireccional a una nueva relación entre lo público y lo privado". Sala de Prensa, 128. En: http://www.saladeprensa.org/art1011.htm [Consulta: 12 de diciembre de 2013].

GRUNIG. James E. and HUNT, Todd (1984): Managing Public Relations. Harcourt Brace, New York, NY.

PEÑALVA, Jorge (2004): "De la comunicación tradicional a la comunicación digital: oportunidades y amenazas". En BELL, Jose Ignacio (Coord): Comunicar para crear valor. La dirección de comunicación en las organizaciones. Barañáin. Ediciones Universidad de Navarra.

PETTIGREW, Justin and REBER, Bryan (2010): “The New Dynamic in Corporate Media Relations: How Fortune 500 Companies Are Using Virtual Press Rooms to Engage the Press". Journal of Public Relations Research, 22 (4), pp. 404-428. http://dx.doi.org/10.1080/10627261003801412

PINEDA MARTÍNEZ, Paula (2013): "Relaciones Públicas Online de excelencia: Las empresas energéticas del IBEX 35 ante el reto de comunicar su conducta responsable en un contexto abierto e interactivo (Mayo-Julio de 2012)" Revista Internacional de Relaciones Públicas, 5 (3), pp. 189-208.

SÁNCHEZ PITA, Fernando y RODRÍGUEZ GORDO, Carlos (2010): “Tendencias en la construcción de salas de prensa virtuales de las principales empresas del índice bursátil IBEX 35" Actas II Congreso Internacional de Comunicación 3.0. Salamanca. pp.1-14.

SORIA IBÁÑEZ, María del Mar (2009):“CComunicación 2.0 y salas de prensa virtuales en las organizaciones no lucrativas españolas". RRPP. Net, portal de Relaciones Públicas. http://www.rrppnet.com.ar/comunicacion2.0.htm [Consulta: 14 de diciembre de 2013]. 